\title{
Modelling and Simulation of Microparticles Separation using Standing Surface Acoustic Waves (SSAWs) Microfluidic Devices for Biomedical Applications
}

\author{
Ahmed M. Soliman \\ Biomedical Engineering \\ Department, Faculty of \\ Engineering, Helwan \\ University, Cairo, Egypt
}

\author{
Mohamed A. Eldosoky \\ Biomedical Engineering \\ Department, Faculty of \\ Engineering, Helwan \\ University, Cairo, Egypt
}

\author{
Taha E. Taha \\ Communication Department, \\ Faculty of Electronic \\ Engineering, Menoufia \\ University, Menouf, Egypt
}

\begin{abstract}
The design of significant and powerful standing surface acoustic wave (SSAW) microfluidic device for microparticles separation for biomedical applications is depending on the dimensions of microchannels for the collecting microparticles. For this purpose, precise calculations of the displacement of microparticles in the working area of SSAW microfluidic device are required. In this paper, the theory and principles of using SSAW microfluidic devices for particles separation are described. The almost published papers in this field had taken into account only the effect of SSAW and viscous drag forces, but the microparticles in the separation process can be affected by other forces. Therefore, the forces acting on the microparticles in fluid flow in SSAW microfluidic devices such as hydrodynamic and diffusion forces are analyzed by mathematical models. Also, the SSAW force is affected by Rayleigh angle, therefore, the SSAW force with the effect of Rayleigh angle which acting on the microparticles is analyzed by mathematical model. The simulation programs are also built by Matlab program to calculate the displacement of microparticles based on the effect of SSAW and viscous drag forces alone; and based on the effect of SSAW with Rayleigh angle effect, viscous drag, hydrodynamic, and diffusion forces. Two types of microparticles different in size and two types of microparticles different in density are separately simulated to verify these programs and show the effects of this analysis. The analysis and simulation of these effects are positively led to more accurate calculations of the displacement of microparticles in the SSAW microfluidic device.
\end{abstract}

\section{Keywords}

Mathematical Models, Microfluidics, Microparticles Separation, Surface Acoustic Wave Devices.

\section{INTRODUCTION}

The separation of particles is important for many biological and biomedical applications including cell biology, diagnostics, and therapeutics, especially in cancer cell detection, drug screening, and tissue engineering [1-3].

Recently, microfluidic devices are used in many fields especially in medicine, biology, chemistry, and biochemistry. The microfluidics can be defined as a system which uses to manipulate the microsample of fluids at micrometers scales [4]. The powerful of microfluidics are in the integration which uses to develop the lab-on-a-chip systems [5]. There are many methods for particle separation used in microfluidic such as hydrodynamic $[6,7]$, dielectrophoretic $[8,9]$, magnetic $[10$, $11]$ and centrifugal $[12,13]$ methods.

The integration of SAWs in microfluidic devices is positively significant in particles separation especially in biomedical applications. The microparticles separation by SSAW microfluidic is depending on size, density, and compressibility of the microparticles. The separation process by SSAW microfluidic devices has many advantages such as portable, high accuracy and sensitivity, low cost, miniaturization, fasting in time compared to traditional methods, reduction in reagents, consumption in samples, and precise control.

The surface acoustic waves (SAWs) can be defined as mechanical waves which propagate on the surface of an elastic medium with most of its energy concentrated near the substrate surface. The physical phenomenon on which the SAW is based is the piezoelectricity, i.e. the virtue by which certain materials produce an electrical charge when mechanically strained or vice versa. In general, piezoelectric substrates such as Lithium Niobate $\left(\mathrm{LiNbO}_{3}\right)$, Lithium Tantalite $\left(\mathrm{LiTaO}_{3}\right)$, Gallium Arsenide (GaAs), and quartz are used.

The SAW is generated by applying alternating electric signal to the exciting transducer called Interdigital transducer (IDT) which is a metal electrode deposited on the piezoelectric substrate, the generated electric field penetrates the piezoelectric substrate and surface acoustic waves are induced based on piezoelectric coupling. This causes an acoustic wave to be launched propagating away from the IDT in both directions and perpendicular to the IDT fingers. The wavelength is determined by the IDT geometry as a following [14]:

$$
\lambda=4 d
$$

Where: $d$ and $W$ are the finger width and finger length, respectively. Fig. 1 shows the schematic of typical IDT.

The acoustic wave propagation velocity is determined by the acoustic mode, orientation and the type of substrate. The fundamental resonant frequency is related to the acoustic wave velocity as given as a following:

$$
f_{0}=v_{0} / \lambda
$$


Where: $f_{0}, v_{0}$ and $\lambda$ are the unperturbed frequency, the unperturbed velocity and wavelength of SAW, respectively [14]. Depending on the principles of the generation of SAWs and its interaction with the medium, the SAW devices are used as sensors $[15,16]$ or as biosensors for biological medium $[17,18]$.

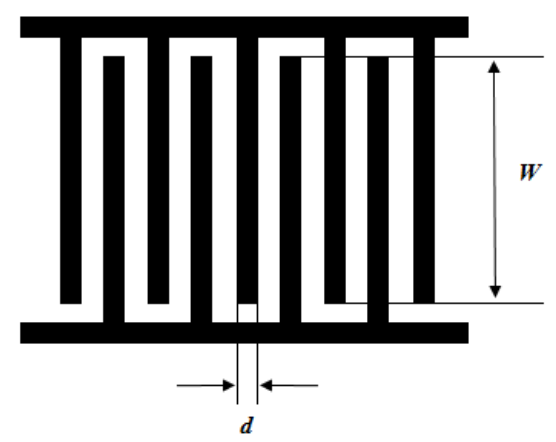

Fig. 1. The schematic of typical IDT, the finger width $(d)$ and finger length $(W)$.

The design of significant and powerful SSAW microfluidic device for microparticles separation is depending on the dimensions of microchannels for the collecting microparticles. For this purpose, precise calculations of the displacement of microparticles in the working area of SSAW microfluidic device are required. In almost published papers in this field had taken into account only the effect of SSAW and viscous drag forces alone such as in [19-21]. These published papers hadn't taken into account the effect of Rayleigh angle on SSAW force; and other forces such as hydrodynamic and diffusion forces.

In this paper, the theory and principles of using SSAW microfluidic devices for particles separation will be described. The mathematical models of the forces acting on the microparticles in fluid flow in SSAW microfluidic devices such as SSAW with Rayleigh angle effect, viscous drag, hydrodynamic, and diffusion forces will be analyzed. Also, the simulation programs are built by Matlab program to show the effect of this analysis compared to the effect of SSAW and viscous drag forces only.

\section{MATHEMATICAL MODELS OF THE FORCES ACTING ON MICROPARTICLES IN FLUID FLOW IN STANDING SURFACE ACOUSTIC WAVES (SSAW) MICROFLUIDIC DEVICES}

The design of significant SSAW microfluidic devices for microparticles separation is most dependently on the calculations of the microparticles displacement in the working area of SSAW force in SSAW microfluidic device. To calculate a more accurate displacement of the microparticles, it should be analysis the different forces acting on the microparticles such as: the acoustic radiation, viscous drag, hydrodynamic, and diffusion forces. Among these forces, buoyancy force are typically balanced by gravity force as they are in opposite directions and almost similar magnitudes, because the densities of both microparticles and the medium are almost equal and in opposite directions. In the following sections, the mathematical models analysis of these forces will be introduced and described.

\subsection{The Acoustic Radiation Force}

The surface acoustic waves can be used for particles separation. This separation is depending on the acoustic radiation force. The two IDTs can generate standing surface acoustic waves (SSAW) to produce acoustic radiation force. The acoustic radiation forces are overlapped and produced pressure node and antinode. These lead the microparticles to migrate toward the pressure node or antinode depending on size, density, and compressibility of the particles. The acoustic radiation force $\left(F_{S S A W}\right)$ is represented as a following [22]:

$$
\begin{gathered}
F_{\text {SSAW }}=-\left(\frac{\pi p_{0}^{2} V \beta_{m}}{2 \lambda}\right) \varphi(\beta, \rho) \sin (2 k x) \\
\varphi(\beta, \rho)=\left(\frac{5 \rho_{p}-2 \rho_{m}}{2 \rho_{p}+\rho_{m}}-\frac{\beta_{p}}{\beta_{m}}\right)
\end{gathered}
$$

Where $p_{0}$ is the acoustic pressure amplitude; $V$ is the volume of the particle; $\lambda$ is the wavelength; $\rho_{p}$ and $\rho_{m}$ are the densities of the particle and the medium, respectively; $\beta_{p}$ and $\beta_{m}$ are the compressibilities of the particle and the medium, respectively; $k$ is the wave number defined by $2 \pi / \lambda$, and $x$ is the distance from the pressure node to the initial particle position; and $\varnothing(\beta, \rho)$ is the acoustic contrast factor. The shape and position of the acoustic wave are depending on the design of SSAW microfluidic device.

The acoustic pressure amplitude can be calculated as a following:

$$
p_{0}=\sqrt{\frac{P Z}{A}}
$$

Where: $P, Z$ and $A$ are the AC signal Power applied to IDT, the acoustic impedance of the piezoelectric substrate and working area of SAW, respectively. The acoustic impedance of the piezoelectric substrate can be calculated as a following:

$$
Z=\rho_{s} v_{0}
$$

Where: $\rho_{s}$ is the density of the piezoelectric substrate.

The acoustic contrast factor $\emptyset(\beta, \rho)$ determines whether the particle will move towards the pressure node or the pressure anti-node. If $\emptyset(\beta, \rho)>0$, the particles will be moved to the pressure node. If $\varnothing(\beta, \rho)<0$, the particles will be moved to the pressure antinode. The biological cells and the solid microparticles in the liquid medium move toward pressure node [23].

In Fig. 2, the schematic of standing surface acoustic wave microfluidic device is shown. In this schematic, the particles mixture is flowed in an inlet microchannel among symmetric side microchannels have sheath fluid (Distilled water). According to SSAW force, the particles are migrated to the pressure node or antinode dependant on its acoustic contrast factor sign. Then, the particles are collected by collecting microchannels according to its physical properties.

As shown in Fig. 3, when SAW propagated on the surface of piezoelectric substrate and entered to liquid medium with an angle called Rayleigh angle $\left(\theta_{R}\right)$ caused by the changing of SAW velocity and can be calculated as a following:

$$
\theta_{R}=\sin ^{-1}\left(\frac{v_{L}}{v_{0}}\right)
$$

Where: $v_{L}$ is SSAW velocity in the liquid medium. 


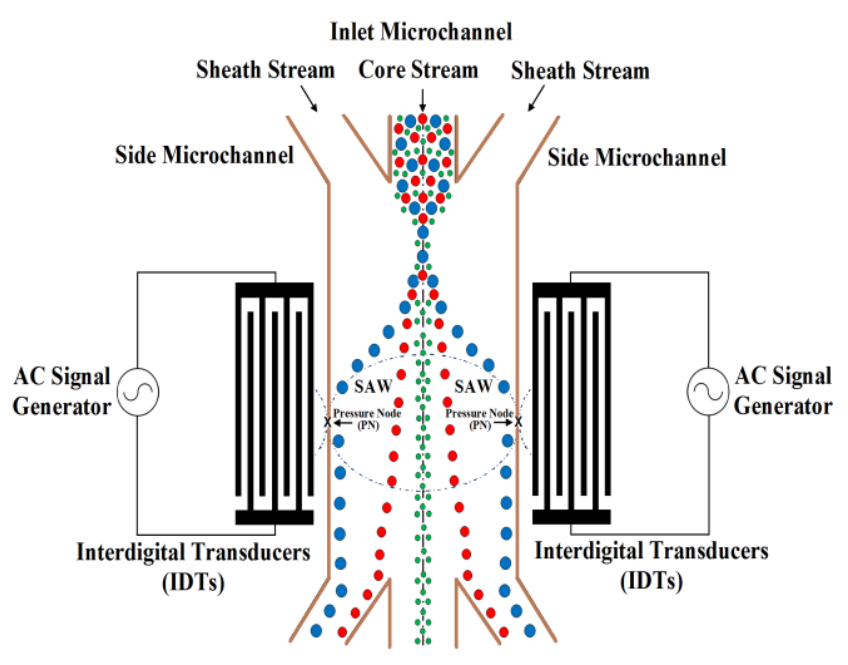

Collecting Microchannels

Fig. 2. The schematic of standing surface acoustic wave microfluidic device.

The acoustic radiation force $\left(F_{S S A W}\right)$ will be affected by Rayleigh angle $\left(\theta_{R}\right)$, then (3) can be modified as a following:

$$
F_{\text {SSAW with AngleEffect }}=-\left(\frac{\pi p_{0}^{2} V \beta_{m}}{2 \lambda}\right) \varphi(\beta, \rho) \sin (2 k x) \sin \left(\theta_{R}\right)
$$

Where: $F_{S S A W}$ with Angle Effect is the acoustic radiation force with Rayleigh angle effect. This modified SSAW force will be led to more accurate calculations for the displacement of the microparticles.

The acoustic radiation force is a combination of the primary and secondary radiation forces. The primary radiation force is produced by SSAW. The secondary radiation force is caused by the scattered acoustic waves from the microparticles [23]. The primary radiation force is more affecting on the microparticles than the secondary radiation force; therefore the secondary radiation force is neglected in this analysis.

\subsection{The Viscous Drag Force}

The viscous drag force is resistive force acting on particles in the opposite direction of acoustic radiation force. The viscous drag force is derived from Stok's law which describes the motion of a sphere in a viscous fluid at a specific velocity.

The viscous drag force $\left(F_{v}\right)$ is given by Stoke's law as a

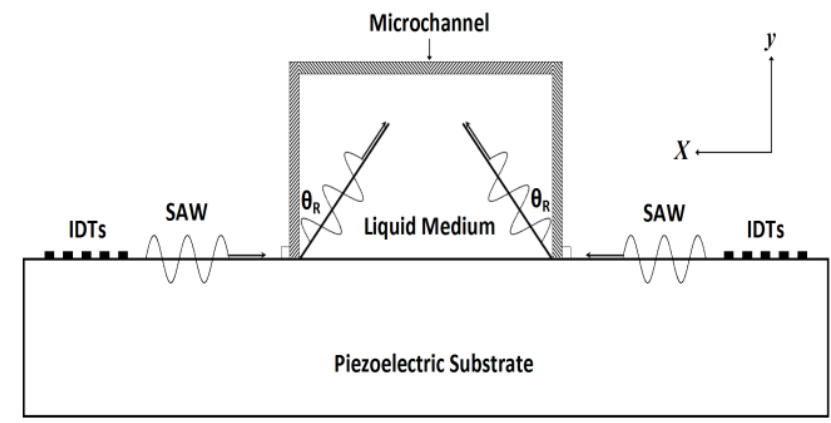

Fig. 3. The configuration of Rayleigh angle $\left(\theta_{R}\right)$ effect on the propagation of surface acoustic wave (SAW). following [24]:

$$
F_{v}=6 \pi \eta r v
$$

Where: $\eta, v$, and $r$ are the fluid viscosity, the relative velocity between particles and medium, and the radius of the particle, respectively.

The acoustic radiation force is dominant compared to the viscous drag force. Also, the viscous drag force can't be neglected. The particles will have net forces proportional to the radius of the particle $(r)$, because the acoustic radiation force is proportional to the $r^{3}$, while the viscous drag force is proportional to $r$. Therefore, the larger particles will move towards the pressure node faster than smaller ones.

When the microparticles have constant velocity in the SSAW field, the acoustic and viscous forces are balanced [25]. According to (3), (4), and (9), the relative velocity of particles to the medium can be expressed as a following:

$$
v=-\left(\frac{p_{0}^{2} V \beta_{m}}{12 \lambda \eta r}\right) \varphi(\beta, \rho) \sin \left(\frac{4 \pi x}{\lambda}\right)
$$

Rewrite $v=-d x / d t$, then (10) can re-write as a following:

$$
\operatorname{cosec}\left(\frac{4 \pi x}{\lambda}\right) d x=\left(\frac{p_{0}^{2} V \beta_{m}}{12 \lambda \eta r}\right) \varphi(\beta, \rho) d t
$$

The displacement of the particle $x$ will be in the range of $(0$, $\lambda / 4)$. By solving (11), the time required to displacement of the particle to pressure node based on SSAW and viscous drag forces only $\left(t_{\mathrm{SSAW}}\right)$ can be expressed as a following [25]:

$$
t_{S S A W}=\left(\frac{3 \lambda^{2} \eta r}{\pi}\right) \frac{\left[\ln \left(\tan \left(\frac{2 \pi x}{\lambda}\right)\right)\right]_{X 1}^{X 2}}{p_{0}^{2} V \beta_{m} \varphi(\beta, \rho)}
$$

Equation (12) can be modified to express the time required to displacement of the particle to pressure node based on SSAW with Rayleigh angle effect and viscous drag forces $\left(t_{S S A W}\right.$ with Angle Effect) can be expressed as a following:

$$
t_{\text {SSAW with AngleEffect }}=\left(\frac{3 \lambda^{2} \eta r}{\pi}\right) \frac{\left[\ln \left(\tan \left(\frac{2 \pi x}{\lambda}\right)\right)\right]_{X 1}^{X 2}}{p_{0}^{2} V \beta_{m} \varphi(\beta, \rho) \sin \left(\theta_{R}\right)}
$$

\subsection{The Hydrodynamic Focusing Force}

The technique of the hydrodynamic focusing in a microfluidic channels can be occurred when a core stream is flowed in an inlet microchannel among symmetric side microchannels have flowed sheath streams. The core stream will be focused in the center of outlet microchannel by sheath streams. The most common configuration is 3-inlet microchannels, one inlet microchannel in a center for core stream and two side microchannels for sheath streams.

When the core stream in the inlet microchannel has microparticles, it is important to determine the width of the focused flow based on hydrodynamic focusing. Thereby, it can be calculate the maximum displacement of microparticles from the center of the outlet microchannel based on the hydrodynamic focusing technique. The width and position of the focused stream can be determined by the relative volumetric flow rates of the three inlets. 
The hydrodynamic focusing flow is shown in Fig. 4. The assumptions used to determine the width of the focused stream $\left(W_{f}\right)$ in rectangular microchannels are the fluids in the microchannels have almost the same densities and Newtonian, the fluid flow is laminar and steady, and the microchannels have the same height [26].

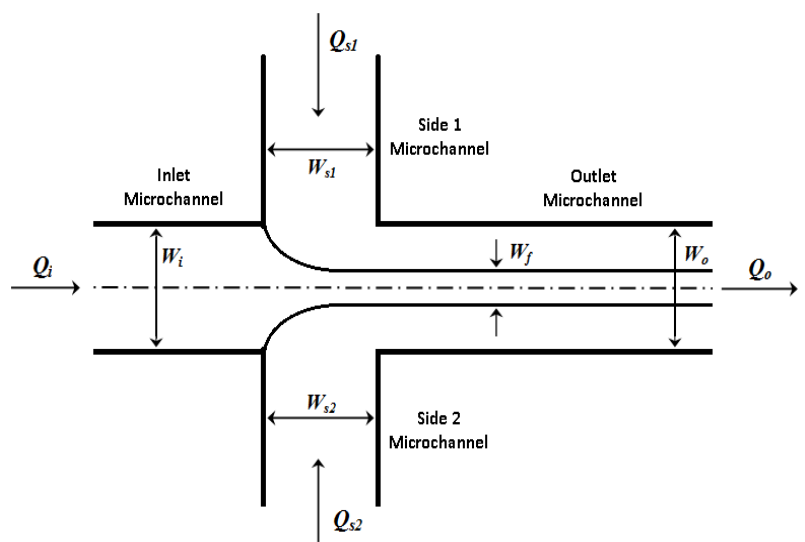

Fig. 4. The hydrodynamic focusing flow.

According to mass conservation law, the width of the focused stream $\left(W_{f}\right)$ can be calculated as a following [26]:

$$
W_{f}=\frac{W_{o} Q_{i}}{\gamma\left(Q_{i}+Q_{S 1}+Q_{S 2}\right)}
$$

Where: $Q_{i}, Q_{s l}, Q_{s 2}, W_{o}, \gamma$ are the volumetric flow rate of the inlet microchannel, the volumetric flow rate of the side microchannels 1 and 2, the width of the outlet microchannel, and the velocity ratio of the average velocity of the focused stream to the average velocity of the stream in the outlet microchannel which assumed as a unity [27].

The width of the focused stream can be easily predicted using (14). This leads to calculate the maximum displacement of microparticles from the center of the outlet microchannel based on the hydrodynamic focusing technique.

\subsection{The Diffusion Force}

The diffusion is the movement of the solute from high to low concentrations of the solute in the solvent [28]. The microparticles in the fluid are affected by the diffusion force and it will be move based on this force. In general, a small particle diffuses faster than a larger one [29].

According to the simple constant-step random walk model of diffusion, the relationship between the diffusion length and time can be expressed as a following [28]:

$$
d_{\text {Diffusion }}=\sqrt{D t_{\text {Diffusion }}}
$$

Where: $d_{\text {Diffusion }}, t_{\text {Diffusion }}, D$ are the diffusion length, diffusion time, and diffusivity, respectively.

\section{DISPLACEMENTS OF MICROPARTICLES BASED ON DIFFERENT FORCES IN STANDING SURFACE ACOUSTIC WAVES MICROFLUIDIC DEVICES}

As described in the previous section, the forces acting on microparticles in the working area of SSAW force in SSAW microfluidic device such as: the acoustic radiation, viscous drag, hydrodynamic, and diffusion forces are analyzed. The displacement of microparticles based on these forces can be calculated as a following:

The displacement of microparticles $\left(d_{S S A W}\right)$ based on the SSAW and viscous drag forces can be approximately expressed according to (3) and (9) as a following:

$$
\begin{gathered}
d_{S S A W}=\frac{\lambda}{2 \pi} \tan ^{-1}\left(e^{c}\right) \\
c=t_{W}\left(\frac{p_{0}^{2} V \beta_{m}}{3 \lambda^{2} \eta r / \pi}\right)+\ln \left(\tan \left(\frac{2 \pi x}{\lambda}\right)\right)
\end{gathered}
$$

Where: $t_{w}$ is the working time of the acoustic radiation force on the microparticle.

The displacement of microparticles ( $d_{S S A W}$ with Angle Effect $)$ based on SSAW with Rayleigh angle effect and viscous drag forces can be approximately expressed according to (8) and (9) as a following:

$$
\begin{gathered}
d_{\text {SSAW with AngleEffect }}=\frac{\lambda}{2 \pi} \tan ^{-1}\left(e^{g}\right) \\
g=t_{W}\left(\frac{p_{0}^{2} V \beta_{m} \sin \left(\theta_{R}\right)}{3 \lambda^{2} \eta r / \pi}\right)+\ln \left(\tan \left(\frac{2 \pi x}{\lambda}\right)\right)
\end{gathered}
$$

Also the maximum displacement of microparticles $\left(d_{\text {Hydrodynamic }}\right)$ from the center of the outlet microchannel based on the hydrodynamic force can be calculated according to (14) as a following:

$$
d_{\text {Hydrodynamic }}=\frac{W_{f}}{2}
$$

The displacement of microparticles $\left(d_{\text {Diffusion }}\right)$ based on the diffusion force was being expressed as in (15).

The total displacements of the microparticles $\left(d_{\text {Total }}\right)$ based on the acoustic radiation with Rayleigh angle, viscous drag, hydrodynamic, and diffusion forces can be approximately expressed as a following:

$$
d_{\text {Total }}=d_{\text {SSAW withAngleEffect }}+d_{\text {Hydrodynamic }}+d_{\text {Diffusion }}
$$

This analysis of the approximate calculation of the total displacement of the microparticles in the fluid flow in surface acoustic wave microfluidics can lead to more accurate imagination about this movement of the microparticles.

\section{RESULTS AND DISCUSSIONS}

The simulation programs are built by Matlab program to calculate the displacement of microparticles based on SSAW and viscous drag forces alone according to (16), and based on SSAW with Rayleigh angle effect, viscous drag, hydrodynamic, and diffusion forces according to (21).

This simulation is based on the schematic of standing surface acoustic wave microfluidic device which shown in Fig. 2. The configuration details used in this simulation is collected from [30]. The width and height of the main channel are 120 and 25 $\mu \mathrm{m}$, respectively. The lengths of the main channel and IDTs are $15 \mathrm{~mm}$ and $9 \mathrm{~mm}$, respectively. Each IDT has 20 electrode finger pairs with $300 \mu \mathrm{m}$ finger pitch and $75 \mu \mathrm{m}$ finger width, corresponding to a SAW wavelength of $\lambda=300 \mu \mathrm{m}$. The SAW substrate is $128^{\circ}$ rotated Y-cut X-propagating lithium niobate (LiNbO3) piezoelectric substrate and its SAW velocity is approximately $3900 \mathrm{~m} / \mathrm{s}$. The AC input power 


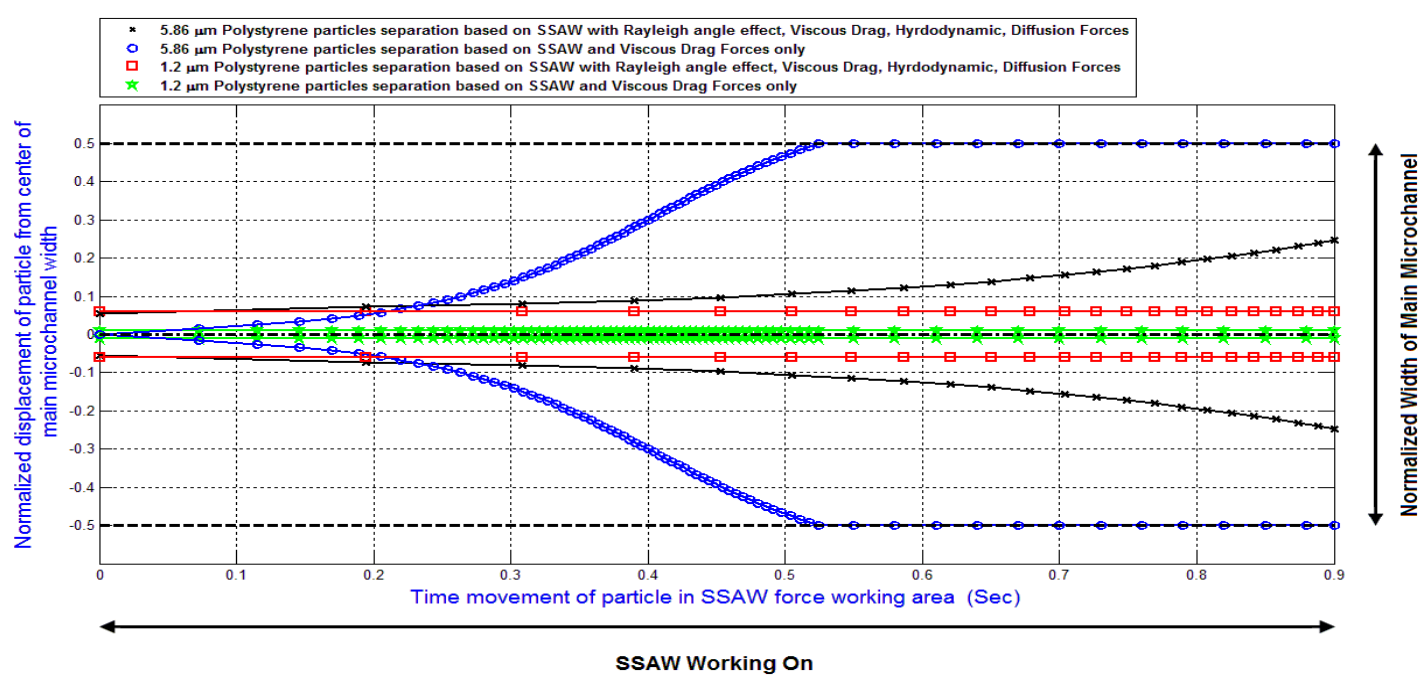

Fig. 5. The separation of two different sizes (1.2 $\mu \mathrm{m}$ and $5.86 \mu \mathrm{m}$ in diameters) of polystyrene microparticles (the same density) based on SSAW and viscous drag forces alone, and based on SSAW with Rayleigh angle effect, viscous drag, hydrodynamic, and diffusion forces at $240 \mathrm{~mW}$ input power.

applied on IDTs to generate SSAWs is about $240 \mathrm{~mW}$. The flow rates of the mixture solution contain microparticles in the middle microchannel inlet and sheath flow (Distilled water) in each of two symmetric side microchannels are $0.2 \mu \mathrm{L} / \mathrm{min}$ and $0.8 \mu \mathrm{L} / \mathrm{min}$, respectively. According to the flow rates of mixture solution and sheath flow, the time required for microparticles to pass through the working area of SSAW force is about $0.9 \mathrm{~s}$.

The mixture solution contains microparticles will be focused in the midline of the main microchannel by the sheath flow (distilled water) in two symmetric inlet side microchannels. This is based on the effect of the hydrodynamic force. Also, the diffusion force will be acted on the microparticles in the focused mixture solution to diffuse from the higher concentration in the mixture solution to the lower concentration in sheath flow.

The SSAW force will be acted on the microparticles in the working area of SSAW force. Therefore, the microparticles will be moved toward the pressure node of SSAW near to the wall of the main microchannel. The displacement of the microparticles will be depended on the physical properties of the microparticles.
The results of these simulations will be represented in two sections. In the first section will be represent the simulation of separation of two different sizes $(1.2 \mu \mathrm{m}$ and $5.86 \mu \mathrm{m}$ in diameters) of polystyrene microparticles (the same density). In the second section will be represent the simulation of separation of the different density microparticles (polystyrene and melamine microparticles) with the same size $(5.86 \mu \mathrm{m}$ in diameters). These simulations will be show the effects of the analysis and verify its programs.

\subsection{The Simulation of Separation of Two Different Sizes of Polystyrene Microparticles (The Same Density)}

The separation of two different sizes $(1.2 \mu \mathrm{m}$ and $5.86 \mu \mathrm{m}$ in diameters) of polystyrene microparticles with density about $1050 \mathrm{~kg} / \mathrm{m}^{3}$ is simulated. This simulation will be represent the displacement of each size of polystyrene microparticles based on SSAW and viscous drag forces alone according to (16), and based on SSAW with Rayleigh angle effect, viscous drag, hydrodynamic, and diffusion forces according to (21).

As shown in Fig. 5 and Fig. 6, the polystyrene microparticles with $5.86 \mu \mathrm{m}$ in diameter are completely moved toward to the

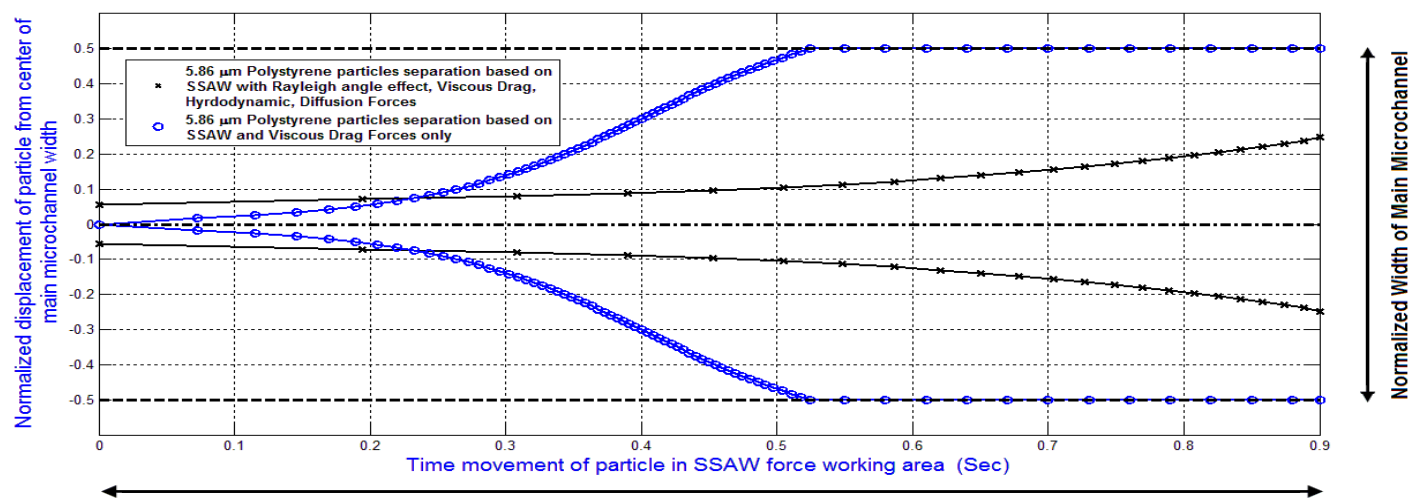

SSAW Working On

Fig. 6. The separation of polystyrene microparticles with $5.86 \mu \mathrm{m}$ in diameter based on SSAW and viscous drag forces alone, and based on SSAW with Rayleigh angle effect, viscous drag, hydrodynamic, and diffusion forces at $240 \mathrm{~mW}$ input power. 


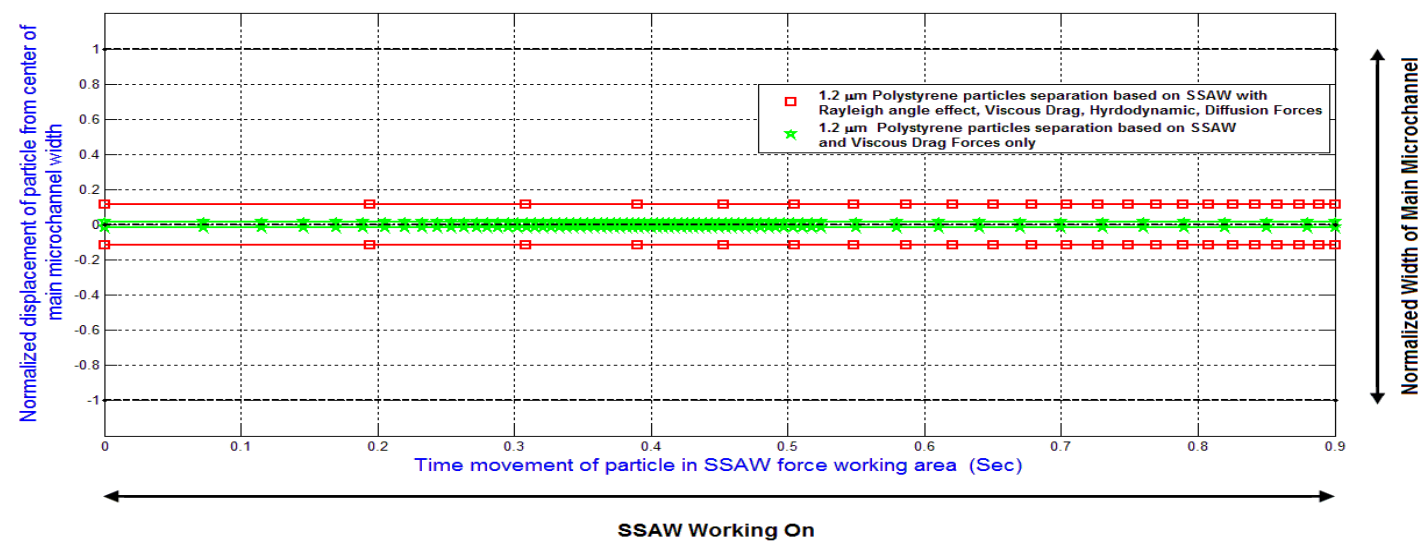

Fig. 7. The separation of polystyrene microparticles with $1.2 \mu \mathrm{m}$ in diameter based on SSAW and viscous drag forces alone, and based on SSAW with Rayleigh angle effect, viscous drag, hydrodynamic, and diffusion forces at $240 \mathrm{~mW}$ input power.

side wall of main microchannel based on the effect of SSAW and viscous drag forces alone after about $0.53 \mathrm{~s}$. On the other hand, based on SSAW with Rayleigh angle effect, viscous drag, hydrodynamic, and diffusion forces, the polystyrene microparticles with $5.86 \mu \mathrm{m}$ in diameter are moved toward to the side wall of main microchannel with about $25 \%$ of the width of main microchannel in the duration of working time of SSAW force. The final displacement of polystyrene microparticles with $5.86 \mu \mathrm{m}$ in diameter based on the SSAW and viscous drag forces only is about twice the final displacement based on SSAW with Rayleigh angle effect, viscous drag, hydrodynamic, and diffusion forces. The interpretation of these results is that the SSAW force is a dominant force to act on the microparticles based on the relatively big size of the microparticles.

As shown in Fig. 5 and Fig. 7, the polystyrene microparticles with $1.2 \mu \mathrm{m}$ in diameter are moved slightly toward to the side wall of main microchannel with about only $1 \%$ of the width of main microchannel in the duration of working time of SSAW force based on the effect of SSAW and viscous drag forces alone. This slight displacement is based on the relatively small size of the microparticles depending on that the SSAW force is proportional to the volume of the microparticles. Therefore, the effect of SSAW force on these microparticles is reduced. On the other hand, based on SSAW with Rayleigh angle effect, viscous drag, hydrodynamic, and diffusion forces, the polystyrene microparticles with $1.2 \mu \mathrm{m}$ in diameter are moved toward to the side wall of main microchannel with about $6 \%$ of the width of main microchannel in the duration of working time of SSAW force. This is based on that the hydrodynamic force is a dominant force. The final displacement of polystyrene microparticles with $1.2 \mu \mathrm{m}$ in diameter based on SSAW with Rayleigh angle effect, viscous drag, hydrodynamic, and diffusion forces is about six times of the final displacement based on the SSAW and viscous drag forces only.

\subsection{The Simulation of Separation of the Different Density Microparticles (Polystyrene and Melamine Microparticles) with the Same Size}

The simulation of separation of the same size $(5.86 \mu \mathrm{m}$ in diameter) of polystyrene with density $1050 \mathrm{~kg} / \mathrm{m}^{3}$ and melamine with density $1710 \mathrm{~kg} / \mathrm{m}^{3}$ microparticles is simulated. This simulation will be represent the displacement of each density of these microparticles based on SSAW and viscous drag forces alone according to (16), and based on SSAW with Rayleigh angle effect, viscous drag, hydrodynamic, and diffusion forces according to (21).

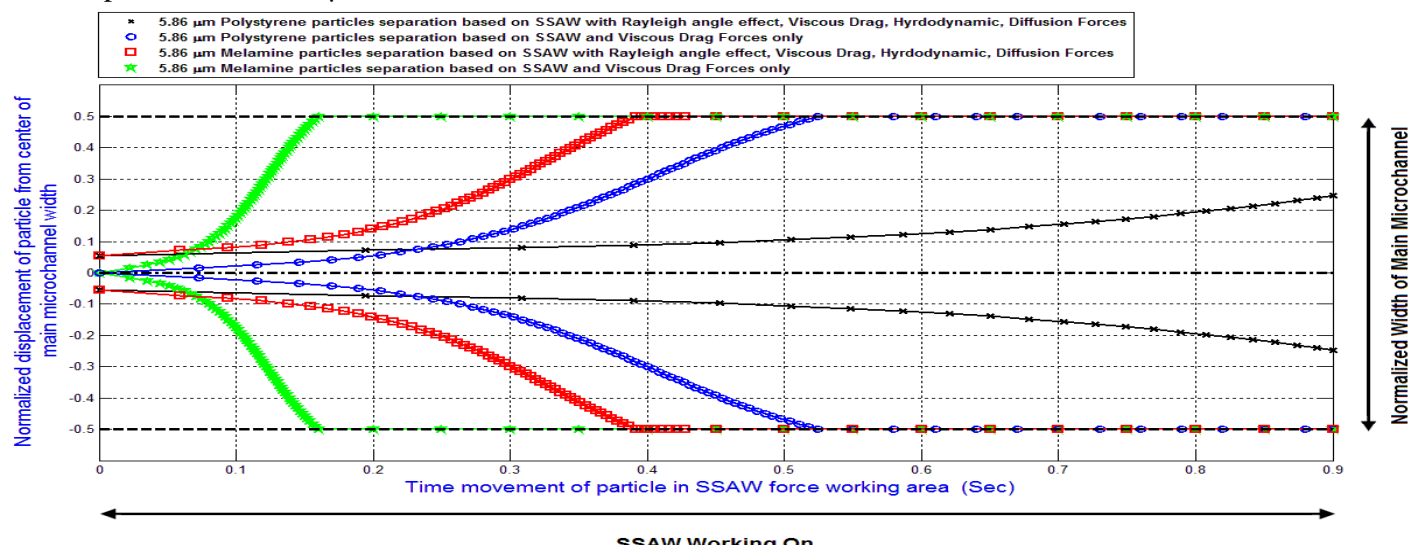

Fig. 8. The separation of the same size $\left(5.86 \mu \mathrm{m}\right.$ in diameter) of polystyrene with density $1050 \mathrm{~kg} / \mathrm{m}^{3}$ and melamine with density $1710 \mathrm{~kg} / \mathrm{m}^{3}$ microparticles based on SSAW and viscous drag forces alone, and based on SSAW with Rayleigh angle effect, viscous drag, hydrodynamic, and diffusion forces at $240 \mathrm{~mW}$ input power. 


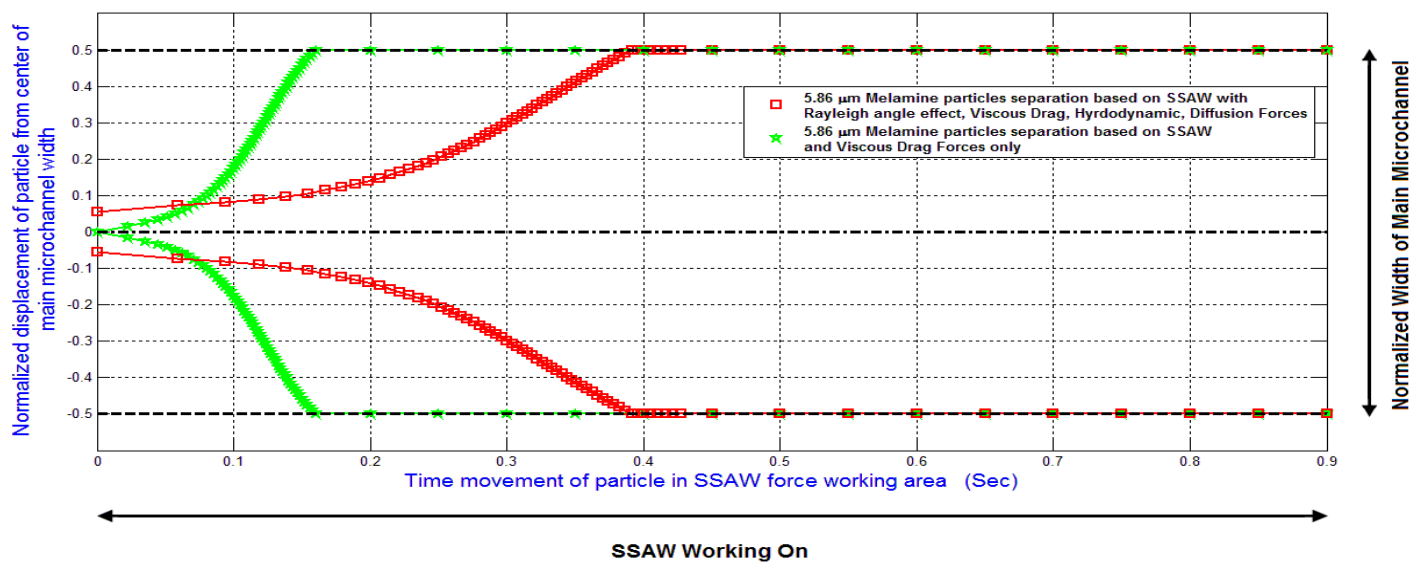

Fig. 9. The separation of melamine with $5.86 \mu \mathrm{m}$ in diameter and $1710 \mathrm{~kg} / \mathrm{m}^{3}$ in density microparticles based on SSAW and viscous drag forces alone, and based on SSAW with Rayleigh angle effect, viscous drag, hydrodynamic, and diffusion forces at $240 \mathrm{~mW}$ input power.

As shown in Fig. 8 and Fig. 9, the melamine microparticles with $5.86 \mu \mathrm{m}$ in diameter and $1710 \mathrm{~kg} / \mathrm{m}^{3}$ in density are completely moved toward to the side wall of main microchannel based on the effect of SSAW and viscous drag forces alone after about $0.16 \mathrm{~s}$. On the other hand, based on SSAW with Rayleigh angle effect, viscous drag, hydrodynamic, and diffusion forces, these microparticles are completely moved toward to the side wall of main microchannel after about $0.39 \mathrm{~s}$. The melamine microparticles with $5.86 \mu \mathrm{m}$ in diameter and $1710 \mathrm{~kg} / \mathrm{m}^{3}$ in density are reached to the side wall of main microchannel based on the SSAW and viscous drag forces only faster about two times and half than based on SSAW with Rayleigh angle effect, viscous drag, hydrodynamic, and diffusion forces. The interpretation of these results is that the SSAW force is a dominant force to act on the microparticles based on its density and size.

As shown in Fig. 8 and Fig. 6, the final displacement of polystyrene microparticles with $5.86 \mu \mathrm{m}$ in diameter and 1050 $\mathrm{kg} / \mathrm{m}^{3}$ in density based on the SSAW and viscous drag forces only is about twice the final displacement based on SSAW with Rayleigh angle effect, viscous drag, hydrodynamic, and diffusion forces. The SSAW force is a dominant force to act on the microparticles based on its density and size.

The movement of microparticles is dependent on its density. Therefore, the movement of melamine microparticles with $5.86 \mu \mathrm{m}$ in diameter and $1710 \mathrm{~kg} / \mathrm{m}^{3}$ in density is faster than the movement of polystyrene microparticles with $5.86 \mu \mathrm{m}$ in diameter and $1050 \mathrm{~kg} / \mathrm{m}^{3}$ in density.

These results are demonstrated the contrast between the calculations based on the analyzed mathematical models and the calculations based on the SSAW and viscous drag forces only. The calculations based on the SSAW and viscous drag forces only are used in almost published papers in the field of size and density dependant microparticles separation based on SSAW microfluidic devices. These published papers had errors in the separation of microparticles. The source of these errors was from the inaccurate calculations of microparticles displacement. This led to the insufficient dimensions of collecting microchannels. They covered these errors by increasing the AC input power to generate stronger SSAW force. Thereby, they assured that the relatively big sizes or high density microparticles will be shifted to the side wall of main microchannel. Therefore, these microparticles will be approximately collected in the two side outlet collecting microchannels.

This simulation program can be used to give an excellent overview about the separation process depending on the effect of acoustic radiation, viscous drag, hydrodynamic, and diffusion forces. This simulation program can be assisted to design accurate and significant standing surface acoustic waves (SSAWs) microfluidic devices used in the separation of microparticles especially in biomedical applications.

\section{CONCLUSION}

The theory and principles of using SSAW microfluidic devices for particles separation are described. The SSAW force with the effect of Rayleigh angle, viscous drag, hydrodynamic, and diffusion forces affected on the microparticles in the separation process are analyzed by mathematical models. The analysis of these effects is positively led to more accurate calculations of the displacement of microparticles in the working area of SSAW force in the SSAW microfluidic device.

The simulation programs are built by Matlab program to calculate the displacement of microparticles based on the effect of SSAW and viscous drag forces alone, and based on the effect of SSAW force with Rayleigh angle effect, viscous drag, hydrodynamic, and diffusion forces. The separation of two different sizes $(1.2 \mu \mathrm{m}$ and $5.86 \mu \mathrm{m}$ in diameters $)$ of polystyrene microparticles (the same density) is simulated. Also, the separation of the same size of polystyrene and melamine microparticles (the different density) is simulated. These simulations are verified these programs and shown the effects of this analysis.

The results of this simulation are demonstrated the contrast between the calculations of microparticles separation based on the analyzed mathematical models and based on the SSAW and viscous drag forces only. The calculations based on the SSAW and viscous drag forces only are used in almost 
published papers in the field of size and density dependant microparticles separation using SSAW microfluidic devices. These published papers had errors in the separation of microparticles. The source of these errors was from the inaccurate calculations of microparticles displacement. This led to the insufficient dimensions of outlet collecting microchannels. They covered these errors by increasing the AC input power to generate stronger SSAW force. Thereby, they assured that the relatively big sizes or high density microparticles will be shifted to the side wall of main microchannel. Therefore, these microparticles will be approximately collected in the two side outlet collecting microchannels.

These simulation programs can provide more accurate calculations of microparticles displacement and lead to design significant and powerful SSAW microfluidic devices for microparticles separation, especially in biomedical and biological applications.

\section{REFERENCES}

[1] M. Hejazian, W. Li, and NT. Nguyen, 2015, "Lab on a Chip for Continuous-Flow Magnetic Cell Separation" Lab on Chip, vol. 15, pp. 959-970.

[2] M. G. Lee, J. H. Shin, C. Y. Bae, S. Choi, and JK. Park, 2013, "Label-Free Cancer Cell Separation from Human Whole Blood Using Inertial Microfluidics at Low Shear Stress" Anal. Chem., vol. 85, pp. 6213-6218.

[3] T. Baciu, F. Borrull, C. Aguilar, and M. Calull, 2015, "Recent Trends in Analytical Methods and Separation Techniques for Drugs of Abuse in Hair" Analytica Chimica Acta, vol. 856, pp. 1-26.

[4] George M. Whitesides, 2006, "The Origins and The Future of Microfluidics" Nature, vol. 442, pp. 368-373.

[5] Tabeling P., 2005, Introduction to Microfluidics, New York: Oxford Univ. Press.

[6] T. M. Geislinger, S. Chan, K. Moll, A. Wixforth, M. Wahlgren, and T. Franke, 2014, "Label-Free Microfluidic Enrichment of Ring-Stage Plasmodium Falciparum-Infected Red Blood Cells Using Non-Inertial Hydrodynamic Lift" Malaria Journal, vol. 13, pp. 1-8.

[7] A. Karimi, S. Yazdi, and A. M. Ardekani, 2013, "Hydrodynamic Mechanisms of Cell and Particle Trapping in Microfluidics" Biomicrofluidics, vol. 7, pp. $1-23$.

[8] M. Mohammadi, H. Madadi, J. Casals-Terré, and J. Sellarès, 2015, "Hydrodynamic and Direct-Current Insulator-Based Dielectrophoresis (H-DC-Idep) Microfluidic Blood Plasma Separation" Anal. Bioanal. Chem., vol. 407, pp. 4733-4744.

[9] Y. Jia, Y. Ren, and H. Jiang, 2015, "Continuous Dielectrophoretic Particle Separation Using A Microfluidic Device with 3D Electrodes and Vaulted Obstacles" Electrophoresis, vol. 36, pp. 1744-1753.

[10] J. Xia, X. Chen, C.Z. Zhou, Y.G. Li, and Z.H. Peng, 2011, "Development of A Low-Cost Magnetic Microfluidic Chip for Circulating Tumour Cell Capture" IET Nanobiotechnology, vol. 5, pp. 114-120.

[11] S. H. S. Lee, T. A. Hatton, and S. A. Khan, 2011, "Microfluidic Continuous Magnetophoretic Protein Separation Using Nanoparticle Aggregates" Microfluidics and Nanofluidics, vol. 11, pp. 429-438.
[12] A. Lee, J. Park, M. Lim, V. Sunkara, S. Y. Kim, G. H. Kim, M. Kim, and Y. Cho, 2014, "All-in-One Centrifugal Microfluidic Device for Size-Selective Circulating Tumor Cell Isolation with High Purity" Anal. Chem., vol. 86, pp. 11349-11356.

[13] L. Clime, D. Brassard, M. Geissler, and T. Veres, 2015 , "Active Pneumatic Control of Centrifugal Microfluidic Flows for Lab-on-a-Chip Applications" Lab Chip, vol. 15 , pp. 2400-2411.

[14] T. E. Taha, M. A. A. El-Dosoky and A. M. El-Sayed, 2007, "On the Analysis of the Interaction between Surface Acoustic Wave (SAW) and Adjacent Media" The $24^{\text {th }}$ National Radio Science Conference (NRSC), Cairo, Egypt.

[15] M. S. Zaghloul, T. E. Taha, A. H. A. Moustafa, and K. El-Shennawy, 2001, "Modeling and Simulation of Fluid SAW-Sensors under Acoustic Plate Mode Operation" IEEE Transactions on Instrumentation and Measurement, vol. 50, pp. 95-100.

[16] T. E. Taha, M. A. A. El-Dosoky and A. M. El-Sayed, 2009, "Estimation of Mixing Ratios of Two Mixture Solutions Using Shear Horizontal Surface Acoustic Wave (SH-SAW) Sensors" The $26^{\text {th }}$ National Radio Science Conference (NRSC), Cairo, Egypt.

[17] T. E. Taha, M. A. A. El-Dosoky and A. M. El-Sayed, 2009, "The Effect of the Loss Tangent of the Blood and the Glucose on the Perturbation of the Shear Horizontal Surface Acoustic Wave" The $26^{\text {th }}$ National Radio Science Conference (NRSC), Cairo, Egypt.

[18] L. Voiculescu, and A. N. Nordin, 2012, "Acoustic Wave based MEMS Devices for Biosensing Applications" Biosensors and Bioelectronics, vol. 33, pp. 1-9.

[19] M. C. Jo and R. Guldiken, 2012, "Active Density-Based Separation Using Standing Surface Acoustic Waves" Sensors and Actuators A, vol. 187, p.p. 22-27,.

[20] J. Nam, H. Lim, D. Kim, and S. Shin, 2011, "Separation of Platelets from Whole Blood Using Standing Surface Acoustic Waves in A Microchannel" Lab on Chip, vol. 11, pp. 3361-3364.

[21] F. Petersson, L. Aberg, AM. Sward-Nilsson, and T. Laurell, 2007, "Free Flow Acoustophoresis: Microfluidic-Based Mode of Particle and Cell Separation" Anal. Chem., vol. 79, p.p. 5117-5123.

[22] K. Yosioka, and Y. Kawasima, Y., 1955, "Acoustic Radiation Pressure on A Compressible Sphere" Acustica, vol. 5, pp. 167-173.

[23] T. Laurell, F. Petersson and A. Nilsson, 2007, "Chip Integrated Strategies for Acoustic Separation and Manipulation of Cells and Particles" Chem. Soc. Rev., vol. 36, pp. 492-506.

[24] F.M. White, 1999, Fluid Mechanics, McGraw-Hill.

[25] J. Shi, D. Ahmed, X. Mao, S. S. Lin, A. Lawit and T. J. Huang, 2009, "Acoustic Tweezers Patterning Cells and Microparticles Using Standing Surface Acoustic Waves (SSAW)" Lab Chip, vol. 9, pp. 2890-2895.

[26] G. B. Lee, C. C. Chang, S. B. Huang and R. J. Yang, 2006, "The Hydrodynamic Focusing Effect inside Rectangular Microchannels" Journal of Micromechanics and Microengineering, vol. 16, pp. 1024-1032. 
[27] T. Stiles, R. Fallon, T. Vestad, J. Oakey, D. Marr, J. Squier and R. Jimenez, 2005, "Hydrodynamic Focusing for Vacuum-Pumped Microfluidics" Microfluidics and Nanofluidics, vol. 1, p.p. 280-283.

[28] H. Bruus, 2008, Theoretical Microfluidics, Oxford University Press.
[29] B. H. Weigl and P. Yager, 1999, "Microfluidic Diffusion-Based Separation and Detection" Science, vol. 283, p.p. 346-347.

[30] Y. Ai, C. K. Sanders, and B. L. Marrone, 2013, "Separation of Escherichia coli Bacteria from Peripheral Blood Mononuclear Cells Using Standing Surface Acoustic Waves", Anal. Chem., vol. 85, p.p. 9126-9134. 